\title{
DESIGN OF A CUSTOM-MADE CRANIAL IMPLANT IN PATIENTS SUFFERING FROM APERT SYNDROME
}

\author{
Mandolini, Marco (1); Brunzini, Agnese (1); Brandoni Serrani, Eleonora (1); Pagnoni, Mario (2); \\ Mazzoli, Alida (1); Germani, Michele (1) \\ 1: Università Politecnica delle Marche; 2: Università degli Studi di ROMA La Sapienza
}

\begin{abstract}
This study defines a methodological procedure for the design and manufacturing of a prosthetic implant for the reconstruction of a midsagittal bony-deficiency of the skull due to the Apert congenital disorder. Conventional techniques for craniofacial defects reconstruction rely on the mirrored-image technique. When the cranial lesion extends over the midline or in case of bilateral defects, other approaches based on thin plate spline interpolation or constrained anatomical deformation are applied.

The proposed method uses the anthropometric theory of cranial landmarks identification for the retrieval of a template healthy skull, useful as a guide in the successive implant design. Then, anatomical deformation of the region of interest and free-form modelling allow to get the customized shape of the implant. A full bulk and a porous implant have been provided according to the surgeon advises.

The models have been 3D printed for a pre-surgical analysis and further treatment plan. They fulfilled the expectancies of the surgeon thus positive results are predictable.

This methodology results to be reproducible to any other craniofacial defect spanning over the entire skull.
\end{abstract}

Keywords: Additive Manufacturing, Biomedical design, Design process, Implant design, Apert Syndrome

\section{Contact:}

Mandolini, Marco

Università Politecnica delle Marche

Department of Industrial Engineering and Mathematical Sciences

Italy

m.mandolini@univpm.it

Cite this article: Mandolini, M., Brunzini, A., Brandoni Serrani, E., Pagnoni, M., Mazzoli, A., Germani, M. (2019)

'Design of a Custom-Made Cranial Implant in Patients Suffering from Apert Syndrome', in Proceedings of the 22nd International Conference on Engineering Design (ICED19), Delft, The Netherlands, 5-8 August 2019. DOI:10.1017/ dsi.2019.75 


\section{INTRODUCTION AND LITERATURE REVIEW}

Apert syndrome is a genetic condition of acrocephalosyndactyly with a high incidence estimated at 1/65.000 births (Driessen et al., 2017). It is characterized by craniosynostosis, midfacial hypoplasia with exorbitism (the protrusion of the eyeball due to a decreased capacity of the orbital container) resulting in a dished profile, syndactyly of the hands and feet at cutaneous and bony level, symphalangism (fusion of digital phalanges), radiohumeral fusion, and varying degrees of neurocognitive impairment (Breik et al., 2016).

The coronal synostosis prevents the forehead from growing forward resulting in an outward and upward pressure exerted by the brain in correspondence of other sutures resulting in a peaked head and a prominent flat forehead (Figure 1), a condition described as acrobrachycephaly (Breik et al., 2016). The orbits or bony sockets which contain the eyes are very shallow causing a bulging or proptosis.

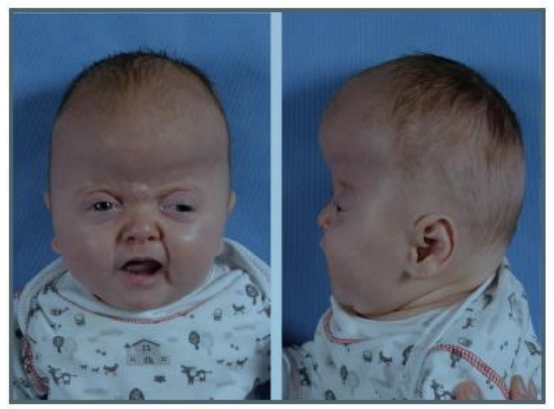

Figure 1. Brachycephaly, exorbitism and midfacial hypoplasia (Driessen et al., 2017)

The traditional approach involves a cranioplasty related to fronto-orbital advancement to increase the intracranial space and size of both orbits. A first step of treatment of this disease is to separate the skull bones that are abnormally fused with one another in a process known as suture release to give a more normal skull shape. Usually, the skull formation surgery is done at the early age of the child, when he/she is between 6 to 8 months old, as the bones are softer and easier to rearrange at this time. Ideal craniofacial implants for facial skeletal augmentation should perfectly fit to the cranial defect minimizing the space between the implant and the bone, aligning the tapered edges with the adjacent bone boundaries (Park et al., 2013). The advent of CAD-CAM technologies replaced the traditional techniques based on manual shaping (Kai et al., 2000) and casting (Bhargava et al., 2010; Dumbrigue et al., 1998), opening the way to new materials for the fabrication of the prostheses and resulting in improved quality and better post-operative outcomes. Customized implants allow to reduce the operation time and to improve the accuracy of the results as the adaptation of the implant's shape and geometry to the patient's anatomy is designed prior to surgery.

Depending on the case-study and the complexity of the cranial lesion, different approaches are available for the reconstruction of cranio-maxillofacial defects:

- $\quad$ Mirrored imaging technique: it is based on the symmetrical nature of bones even if a small amount of asymmetry is always present in the human body. Even if this method has many applications, it is more suitable for the treatment of unilateral skull damage (Brunzini et al., 2017; Drstvensek, 2008).

- $\quad$ Thin plate spline surface (TPS) interpolation or deformation: A TPS deformation visualizes the shape difference between one reference form and one target form, based on a set of homologous point coordinates measured on both forms and minimizes the integral of the squared second derivatives which measures the amount of local shape deformation (Benazzi et al., 2011; Carr et al., 1997; Dean, 2003; Gunz et al., 2013).

- Anatomical reconstruction or free form surface: in case of defects that extend through the midline, it is preferable to adopt the anatomical reconstruction approach by generating curves based on the anatomical region with CAD based/haptic devices. Moiduddin et al. (2016) demonstrated that the anatomical reconstruction technique is more efficient than the mirroring technique in the mandible reconstruction showing less deviation with respect to the reference bone. Bogu et al. (2017) applied the mirror technique and generated a surface patch to properly fill the frontal bone deficiency using profile curves to replicate the missing bone fragment. 
- Anatomically constrained deformation: adaptive deformation is used to design the implant geometry according to the defect area on the patient skull and its corresponding fragment on a reference model. According to Ting Wung et al. (2006) a spatial correspondence between the cranial landmarks on the target skull and a template skull has been performed by 3D matching. The implant surface is the result of deforming the corresponding fragment on the reference model by radial adaptive scaling.

In this work, due to the facial deformities caused by Apert syndrome, we developed a novel procedure, which combines the approaches of anatomical reconstruction and constrained deformation thus assuring a more precise design of the custom-made cranial implant.

The research methodology consisted in firstly exploring the state of art for evaluating current practices and relative limitations (chapter $§ 1$ ). Then, a patient affected by Apert syndrome has been selected for supporting the definition of the proposed procedure as well as for testing it. The procedure, thought for overcoming the limitations arose from the literature review, consisted in multiple steps from computer tomography pictures till the rapid prototyping of the designed implants for their pre-operative evaluation (chapter \$2). At the end, a physical implant has been used for the forehead augmentation of the selected patient (chapter $\$ 3$ ). This step allowed the evaluation of the benefits such procedure guarantees (chapter §4).

\section{MATERIAL AND METHODS}

This section deals with the steps involving the methodology developed for the design of the custommade implant. This work aims to restore a bony defect extended through the midsagittal plane in a pathological patient affected by facial disproportion (Apert syndrome) compromising the application of the mirroring technique. Moreover, it deals with a bony deficiency condition requiring thus a meticulous precision in the design of the cranial implant to fit specifically the anatomical site. For this reason, we developed a different procedure for the design of the implant, using a combined approach of anatomical reconstruction and constrained deformation. A template of not pathological skull has been selected and morphometrically adapted to the patient anatomy, to be used as a guide in the design of the prosthesis, using a 3D modelling tool for free-form reconstruction.

\subsection{Design procedure}

The phases of this design methodology with a computer-assisted approach can be summarized by the workflow in Figure 2.

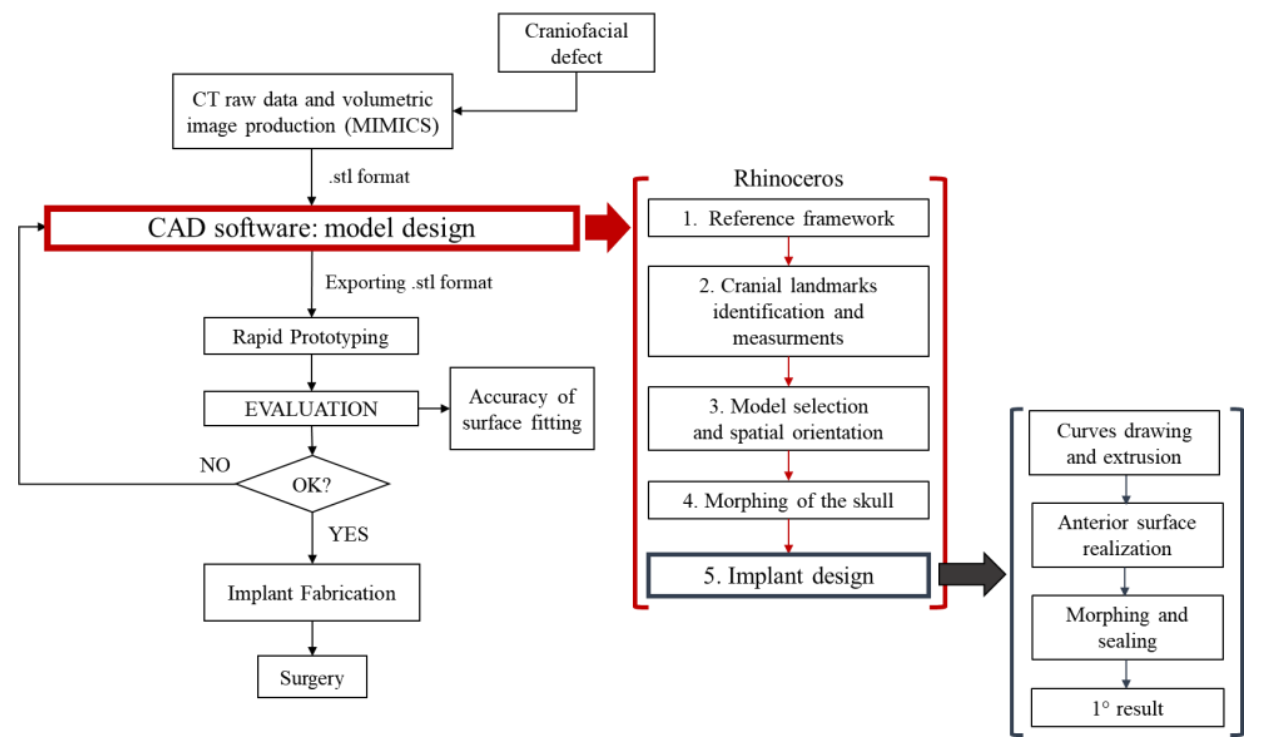

Figure 2. Workflow representing the steps of the customized cranial implant design methodology

With this procedure it is necessary to choose an appropriate existing skull model from a CT (Computer Tomography) database of not pathological skulls: the idea is to reconstruct the missing region of the 
patient's skull (forehead, in this case) following the shape of a healthy person's bone region and adapting its dimension to the patient's anatomy. The main steps can be described as follows:

- Acquisition of CT scan images of the patient's skull, 3D geometric model reconstruction and orientation according to a reference coordinate system (section \$2.2).

- Identification of the main relevant cranial landmarks to compute measurements that provide the information necessary to choose the best matching skull to be used as a template for the implant (section §2.3).

- Selection from a CT database of an appropriate skull to be used as a reference model for the implant design, and spatial orientation of the skull to be overlapped (section §2.4).

- $\quad$ Morphing of the reference skull along the coronal and sagittal plane (section §2.5).

- Implant design: this last step is obtained by isolating the region of interest and realizing the anterior surface of the prosthesis by adapting the curvature of the model of healthy forehead to the requirements of the pathological skull. Then a morphing with smooth transition from the implant to the patient's bony contour is realised (section $\$ 2.6$ ).

\subsection{CT scan acquisition and skull orientation}

The CT images of the patient's skull are acquired in DICOM format to evaluate the affected region. With the medical imaging software MIMICS by Materialise, image processing is performed to separate the bony anatomy from surrounding tissues according to an adequate grey threshold. CT images have been segmented to obtain cross-section views of the skull in axial direction. Then the scan images have been converted into the patient's 3D anatomical skull (Figure 3).
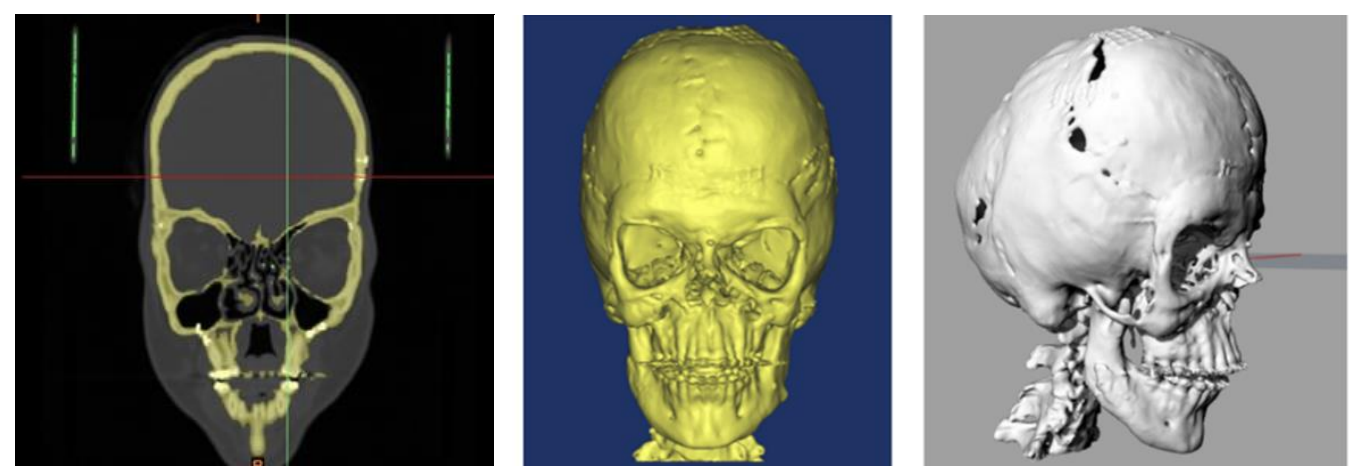

Figure 3. From the left to the right: CT Image of the pathological skull, 3D model reconstruction in Mimics and its representation in Rhinoceros

To achieve useful information by comparing two skulls of different individuals, it is important to have both skulls in the same orientation by aligning the geometry to a reference frame.

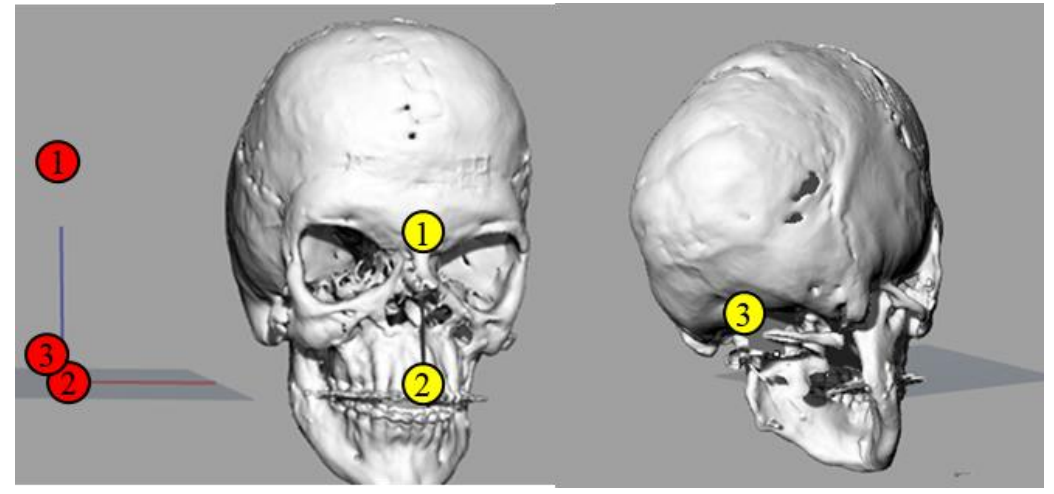

Figure 4. Alignment of the sagittal plane of the skull to the Cartesian YZ plane. Point 1: Nasion, Point 2: Prosthion, Point 3: Opisthion

The procedure provides the creation of a symmetry plane (corresponding to the mid-sagittal plane of the skull) to simplify the overlapping between the two models of skull. The sagittal plane corresponds to the plane of symmetry that vertically divides the skull into two corresponding symmetrical sides. It has been determined through the anthropometric theory based on the localization of cranial landmarks, 
suggested by Farkas (1994). To define this plane three bony unpaired craniometric landmarks have been selected to be far enough to reduce the error in the alignment. The symmetry plane is generated by interpolation of the following points: Nasion, Prosthion, Opisthion (Figure 4). This plane is used to overlap the sagittal plane of the skull to the sagittal YZ Cartesian plane.

\subsection{Cranial landmarks identification and measurements}

A CT scans database of twenty skulls with healthy morphology has been collected and indexed about the demographic information (e.g. age, sex, height) of the subjects. To select the most compatible skull to be used as a model for the implant, it is necessary to identify the most significant cranial references, among those defined in literature and anatomical atlases, to obtain the measurements that provided the information for the selection algorithm (Table 1). According to literature and anatomical atlases, a set of 46 cranial landmarks and 37 measurements is enough for characterizing the morphology of the head.

Although anthropological investigations have found a series of reference points to perform comparable measurements on the skull, it has been impossible to identify the cranial points defined by the coronal and sagittal sutures due to the highly irregular surface of the patient's skull and the surgical operations undergone. Nevertheless, it has been possible to identify, over the pathological skull, the cranial landmarks described in Figure 5, which are the ones that most influence the geometry of the custommade implant to be designed.

Table 1.Cranial landmarks definitions

\begin{tabular}{|l|l|}
\hline LANDMARKS & DEFINITION \\
\hline Maxillofrontal & Anterior lacrimal crest and the frontomaxillary suture intersection point \\
\hline Nasion & The midpoint of nasofrontal suture \\
\hline Orbital & The lowest point in the margin of the orbit \\
\hline Porion & The highest middle point on the margin of the external auditory meatus \\
\hline Prosthion & The median point on the posterior margin of the occipital foramen \\
\hline
\end{tabular}

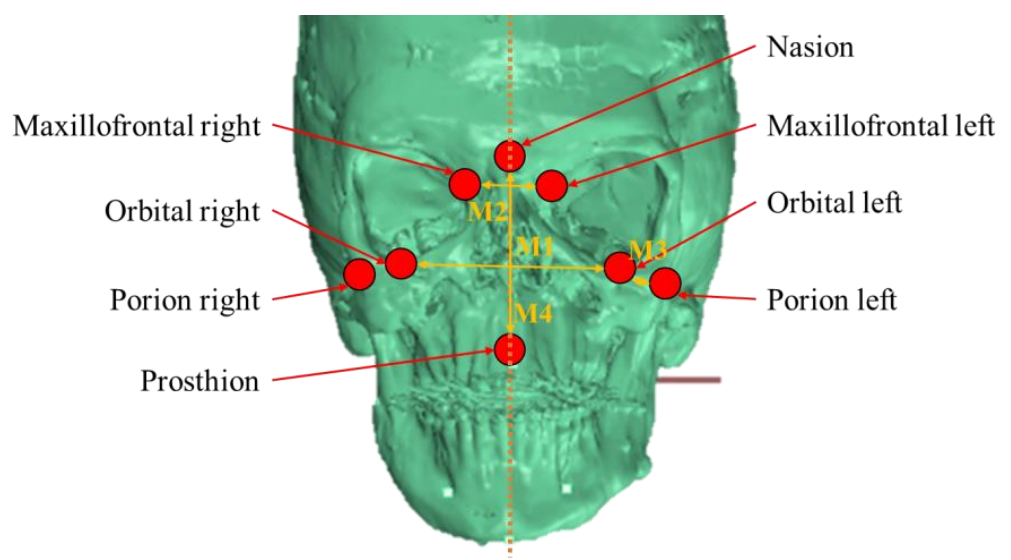

Figure 5. Cranial landmarks identified over the pathological skull

\subsection{Reference model selection and spatial orientation}

The algorithm used to select the appropriate model allows to determine, from a database of samples, the healthy skull with the lowest deviation from the pathological one. The first step of the procedure consists in filtering the healthy skulls according to age and gender. The algorithm is based on the analysis of the measurement's deviations between the pathological and healthy skulls. The healthy model with the lowest deviation from the pathological skull is selected.

Equation (1) is used to assign a score according to the measurements for each skull $(x)$ in the database:

$$
\text { Score }_{-} x=\frac{\sum_{i=1}^{4}\left|\frac{M i x-M i p}{M i p}\right| * p i}{4}
$$


where $i$ refers to each measurement $M . M_{1}$ is the interorbital distance, $M_{2}$ is the inter maxillofrontal distance, $M_{3}$ is the distance between Porion and Orbital and $M_{4}$ the distance between Nasion and Prosthion. $M_{i p}$ stands for the measurements computed over the pathological skull. The database of reference skulls consists of 20 geometries. The score is a weighted mean where the weight is represented by $p i$ and is calibrated according to the importance of the involved measurements. $p 1=p 2$ $=0.33$ as the frontal measurements are more relevant than the last two ones that have one third of the weight hence $p 3=p 4=0.17$. The lowest score corresponds to the smallest measurement deviation from the skull under study, thus it is selected and adapted for better fit on the anatomical region of the patient's skull.

\subsection{Morphing of the reference geometry}

An anatomical deformation process based on scale factors has been performed in order to make the reference model suitable for the pathological skull. The measurements in Table 2 allow to define a spatial correspondence between the cranial landmarks of both the skulls (the patient one and the reference one).

Table 2. Cranial landmarks coordinate of patient's skull and reference skull

\begin{tabular}{|l|c|c|c|c|c|c|}
\hline & \multicolumn{3}{|c|}{ PATIENT } & \multicolumn{3}{c|}{ REFERENCE } \\
\hline LANDMARK COORDINATES [mm] & $\mathbf{X}$ & $\mathbf{Y}$ & $\mathbf{Z}$ & $\mathbf{X}$ & $\mathbf{Y}$ & $\mathbf{Z}$ \\
\hline Right Maxillofrontal & -13.5 & 10.1 & 61.5 & -8.5 & 9.6 & 59.9 \\
\hline Left Maxillofrontal & 13.9 & 9.3 & 61.3 & 8.8 & 9.7 & 60.2 \\
\hline Nasion & 0.0 & 0.0 & 69.4 & 0.0 & 0.0 & 69.4 \\
\hline Right Orbital & -35.1 & 15.0 & 37.6 & -30.4 & 15.7 & 36.9 \\
\hline Left Orbital & 37.3 & 15.7 & 35.3 & 31.5 & 15.7 & 37.5 \\
\hline Right Porion & -47.7 & 75.5 & 33.3 & -51.0 & 87.4 & 44.2 \\
\hline Left Porion & 52.6 & 82.0 & 28.3 & 47.2 & 86.4 & 44.8 \\
\hline Prosthion & 1.8 & -5.1 & 4.8 & 0.0 & 0.0 & 0.0 \\
\hline
\end{tabular}

The morphing procedure consisted in a deformation of the healthy skull on the frontal and sagittal planes by considering respectively measurements M1 and M2 for the first plane and M3 and M4 for the second one. For the refe2081rence skull morphing on the frontal plane, M1 and M2 have been selected because they are the ones that most influence the dimensions of the implant to be designed. Hence, a good superimposition between the reference and pathological skulls on such plane must be achieved. Moreover, for the reference skull morphing on the sagittal plane, M3 and M4 have been selected because these ones are measured on this plane. The scale factors used for frontal and sagittal morphing have been calculated by averaging the ratio between the reference and pathological dimensions, M1 and M2 for the frontal morphing, M3 and M4 for the sagittal morphing (Table 3).

Table 3. Scale factors to morph the reference skull

\begin{tabular}{|c|c|c|c|c|}
\hline MEASUREMENTS & $\begin{array}{c}\text { PATIENT } \\
\text { mm }\end{array}$ & $\begin{array}{c}\text { REFERENCE } \\
\text { mm }\end{array}$ & SCALE FACTOR & $\begin{array}{c}\text { AVG. SCALE } \\
\text { FACTOR }\end{array}$ \\
\hline $\begin{array}{l}\text { M1. Interorbital distance } \\
\text { (frontal plane) }\end{array}$ & 72.4 & 61.9 & 1.17 & \multirow[t]{2}{*}{1.37} \\
\hline $\begin{array}{l}\text { M2. Maxillo-frontal } \\
\text { distance (frontal plane) }\end{array}$ & 27.4 & 17.3 & 1,58 & \\
\hline $\begin{array}{l}\text { M3. Porion-orbital } \\
\text { distance (sagittal plane) }\end{array}$ & $\begin{array}{l}66.7 \text { (left) } \\
60.1 \text { (right) }\end{array}$ & $\begin{array}{l}73.0 \text { (left) } \\
75.6 \text { (right) }\end{array}$ & $\begin{array}{c}0.90 \text { (left), } 0.80 \\
\text { (right), Average: } \\
0.85\end{array}$ & \multirow[t]{2}{*}{0.89} \\
\hline $\begin{array}{l}\text { M4. Nasion-prosthion } \\
\text { distance (sagittal plante) }\end{array}$ & 64.8 & 69.4 & 0.93 & \\
\hline
\end{tabular}

The scale factors applied to the template skull have allowed to overlap the orbital points to those ones of the patient, resulting in a greater matching in the region of interest.

To complete the morphing of the template skull in the forehead region, a third non-uniform scale has been performed using control points of a cage fixed over the reference skull (Figure 6). This further step allows to start designing the patient-specific cranial implant. 


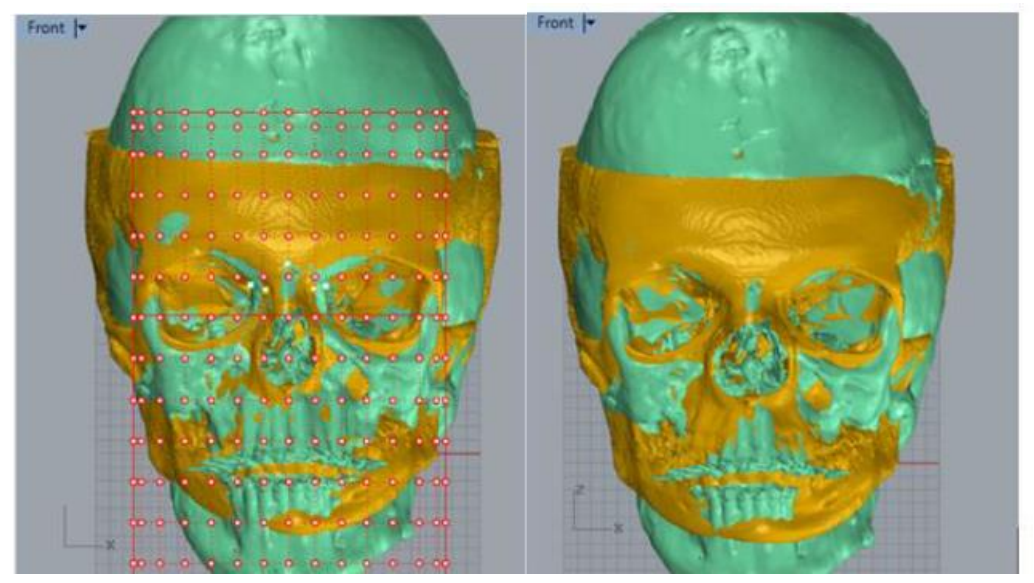

Figure 6. Non-uniform scale to match the maxillo-frontal points. Before the scale (on the left) and after the scale process (on the right)

\subsection{Implant design}

Two slightly different kinds of personalized implant have been realized in Rhinoceros by McNeel: a bulky one (more resistant) and a porous one (which allow a better osseointegration). The steps followed to design the first implant are shown in the flow-chart in Figure 2. Then, from the custommade bulky implant, a porous implant has been designed by adding the porosity to the first one.

The implant design starts with the extrusion of two sets of curves to define a guide for the realization of the anterior and posterior surfaces of the implant. In order to achieve a smooth and continuous transition between the implant and the skull, the border of the implant anterior face is tapered to the posterior one and then morphed at the interface with the skull. With a Boolean union the two implant faces are sealed into one closed object.

In Figure $7 \mathrm{a}$ the profile curves used to define the region of interest are represented. One of these curves tracks the profile of the forehead just over the eyebrow while the second curve defines the extension of the implant until the temples. The region of interest is split and isolated from the rest of the skull and all the compenetrating layers of the same mesh are removed in order to obtain two layers representing the anterior and the posterior side of the implant.
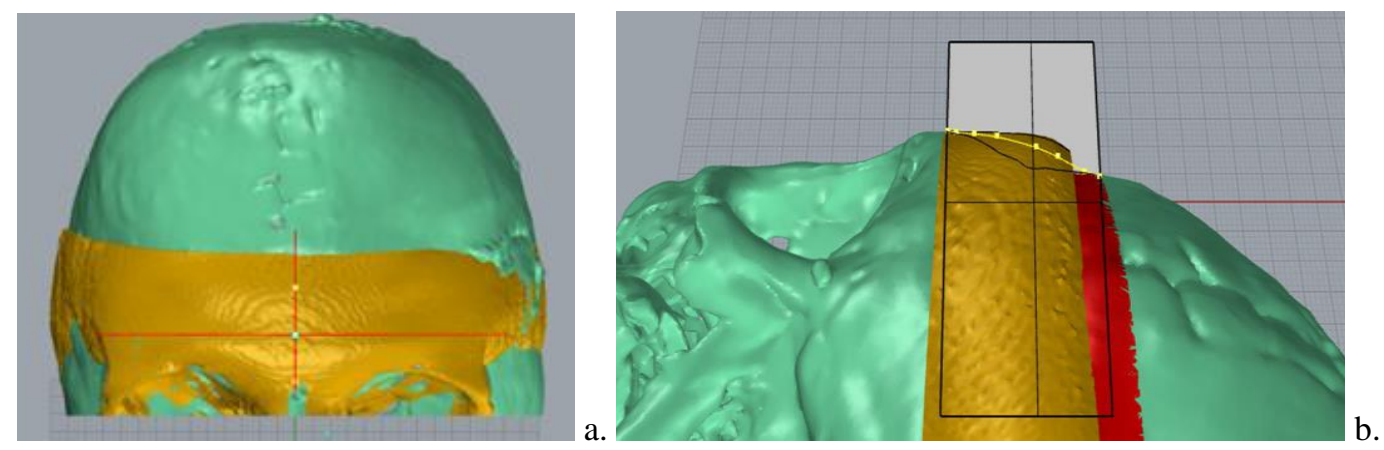

Figure 7. Profile curves (red) used to define the region of interest on the sagittal plane (a); Contour smoothing of the profile curve of the surface by means of control points (yellow)(b)

To create the anterior surface of the implant, 15 radial planes have been defined to intersect perpendicularly with the template forehead along its length, the posterior face of the implant and the patient's skull. Each intersection results in a profile curve. A line has been tracked to define the curvature of the implant and then it has been adjusted, through control points, to be tangent to the borders of the implant ensuring a continuous and smooth transition between the skull and the implant itself (Figure 7b). Moreover, control points on the implant surface have been used to morph and fit the curvature of the surface. The interface between the skull and the implant must be continuous and smooth avoiding any protrusion that would result in unpleasant aesthetic result (Figure 8.a). Then, to improve the process of osteointegration, a porous version of the implant has been designed (Figure 8.b). The porosity has been created by extruding an array of squares into solid parallelepipeds which have been subtracted from the original bulk volume through Boolean operations. The porosity is given 
by blinded-end channels with a square cross-section of $500 \mu \mathrm{m}$. The porosity is limited to the implant posterior-central zone, to avoid unpleasant tactile feeling on the patient forehead and to guarantee an adequate strength to the implant. This allows getting a porosity of $10 \%$, which is in the range of the natural porosity of the cortical bone.
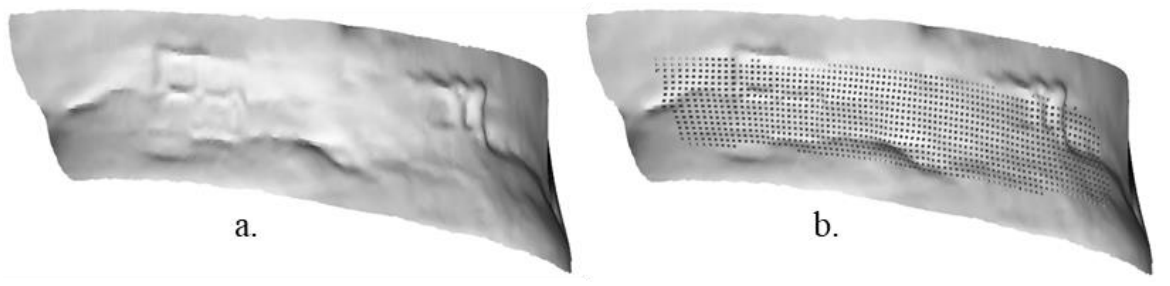

Figure 8. Custom made implants designed for patient suffering from Apert syndrome: full bulk (a) and porous (b) ones

\section{RESULTS AND DISCUSSION}

The results of the proposed methodology, tested on a patient suffering from Apert pathology consisted in two custom-made cranial implants, full bulk volume and porous implant, for forehead augmentation (Figure 9).
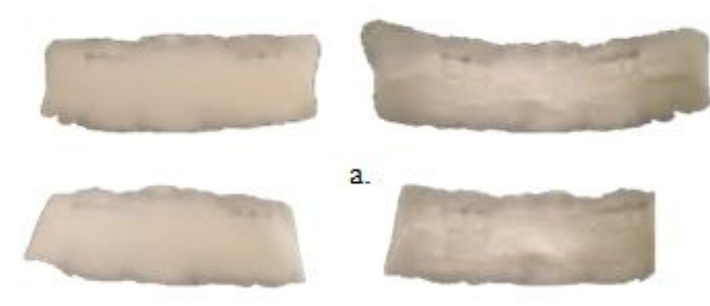

a.

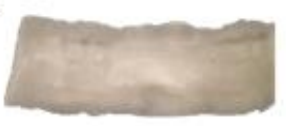

b.

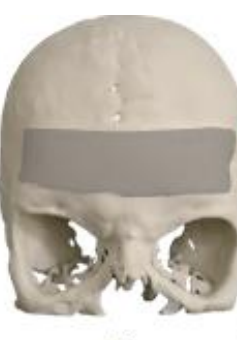

c.

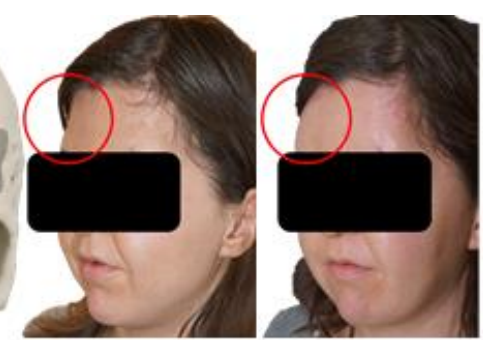

d.

e.

Figure 9. a. Full bulk volume implant front (left) and back side (right); b. Porous implant front (left) and back side (right); c. Custom made implant (full bulk volume) overlapped with patient skull; $d$. Pre-operative patient forehead; $d$. Post-operative patient forehead.

The Apert pathology related defects extend through the skull midline, hence, the application of the mirrored-image technique is not applicable. Moreover, this is about a bony deficiency, thus, there was no bone to be mirrored. This situation led to the retrieval of a template bone to drive the process of forehead reconstruction. A CT dataset of healthy skulls was built up and indexed by labels about age and gender. Measurements taken between cranial landmarks, suggested by anthropometry, allowed to select the most suitable skull to the case under study. This first step of the procedure included some limitations; the size of the CT dataset should be extended in number and updated with a library of cranial regions for a quicker retrieval of the anatomy and use (Milovanović and Trajanovic, 2007). Moreover, an automatic procedure for the extraction of cranial landmarks and geometrical comparison based on the relative measurements could save time and improve the accuracy of the result as up to now it is based on manual selection of landmarks under a subjective perception skill. Software tools as Edgewarp, MorphoJ, tpsDig and Landmark can be useful in this regard as they allow for an easier automatic extraction of landmark coordinates.

Morphing was performed to achieve a full match between the healthy skull and the pathological skull, to adjust the geometry of the model and to seal the two sides that would made the implant to create a closed polysurface ready for the 3D printing. The need for anatomical morphing of the template skull would be useful even if the cranial selection approach was totally automated. Therefore, the step of morphing is thought to be of greatest relevance and unavoidable.

The first result involved a solid, compact prosthesis as represented in Figure 8a, with dimensions $97.53 \times 22.40 \times 30.38 \mathrm{~mm}$ and a variable thickness between $3 \mathrm{~mm}$ at central region and $0.1 \mathrm{~mm}$ at the sides. The implant fixation is realized through four screws placed close to the four corners at a certain angle to reduce visibility. The screw gauge is $1.5 \mathrm{~mm}$ with a length of $2-3 \mathrm{~mm}$ thus should not provide any problem. 
A second proposal of customized implant assumed a certain degree of porosity within the implant for several reasons (Figure 8b). If we consider fabricating the implant in PEEK, porosity can favour osteointegration of the implant as PEEK is biologically inert. Thus, a porosity of $10 \%$ to match cortical bone porosity was considered. The pore size used for most bone ingrowth settings is between 150 and $500 \mu \mathrm{m}$, which is just large enough to support ingrowth of vascular tissues and cellular migration (Muschleret al., 2004; Karageorgiou and Kaplan, 2005). Hence, pores of $500 \mu \mathrm{m}$ were realized to match the porosity of cortical bone. However, this version of the implant was discarded by the surgeon as the forehead of the patient was covered by a metallic mesh thus preventing the exposition of the implant to the natural bone. This meant no possibility of osteointegration. Both the implants and the patient's skull have been $3 \mathrm{~d}$ printed for physically simulating the aesthetical benefits of the proposed implants (Figure 8c).

At last, surgeon implanted the first proposed implant, which has been realized according the procedure described in Mandolini et al. (2019) and using a sheet of Medpor Titan ${ }^{\circledR}$ (composite material of highdensity polyethylene and titanium mesh). The red circle highlights the aesthetical benefits achieved between the pre-operative (Figure 8d) and post-operative conditions (Figure 8e). The forehead, originally flat, has been augmented with the aim to recreate the anatomy/curvature of healthy people.

The procedure here proposed allowed the surgeon to be confident of the results achievable after the surgery. In a traditional approach, the implant is commonly shaped on the patient's skull during the surgery, causing inaccurate implant placement, trauma to tissues and long surgical time (Mandolini et al., 2019). Some steps of the design procedure (i.e. acquisition of CT scan, identification of the main relevant cranial landmarks, selection and morphing of the reference skull from a CT database) can be also automated by developing macros to plug into a 3D CAD system. Furthermore, the adoption of such procedure allowed an approximately $50 \%$ reduction of the surgery duration.

\section{CONCLUSIONS}

Facial deformities and cranial lesions due to congenital disorders, accidents and tumours require accurate and time expensive procedures for aesthetic reconstruction. Custom implants for the reconstruction of craniofacial defects have gained importance since the advances in imaging techniques and CAD-CAM systems allowing for a precise adaptation to the region of implantation, reduced surgical times and better aesthetic outcomes. The aim of this paper was to develop a method for designing a customized prosthesis for forehead augmentation specific for the pathological condition of Apert syndrome.

The presented methodology for the design of the cranial implant is a two-steps procedure based on cranial landmarks identification for the selection of a healthy skull and for mid-sagittal plane definition and a free form morphing for the adaptation of the implant, thus it can be applied to any other cranial deformation without the limitations of the mirroring technique. The procedure has been tested on a patient suffering from Apert pathology with the aim to design two custom-made cranial implants, full bulk volume and porous implant, for forehead augmentation. The implants virtual prototypes have been 3D printed using a polymer powder-based device for better visualization of the result and surgery simulation (Figure 9). Rapid Prototyping and computer modelling improved the surgical planning and the manufacture of the patient-specific implant testing its fit before the actual surgery.

Currently, the landmarks identification, reference skull identification and morphing are done manually resulting in a time-consuming process. A digital landmark identification and extraction based on a combined approach of knowledge based and learning methodologies may improve the efficiency and the reproducibility of the algorithm. Automated macros and scripts, built within a 3D CAD system, should be developed for speeding up the design process. Furthermore, it could be useful to create a library of anatomical bony regions ready for the retrieval of a compatible module to save time.

\section{ACKNOWLEDGMENTS}

Authors would like to acknowledge Eng. Manila Caragiuli for her contribution in this research project. 


\section{REFERENCES}

Benazzi, S. and Senck, S. (2011), "Comparing 3-Dimensional Virtual Methods for Reconstruction in Craniomaxillofacial Surgery”, Journal of Oral Maxillofacial Surgery, Vol. 69, pp. 1184-1194. http://doi.org/10.1016/j.joms.2010.02.028

Bhargava, D., Bartlett, P., Russell, J., Liddington, M., Tyagi, A. and Chumas, P. (2010), "Construction of titanium cranioplasty plate using craniectomy bone flap as template", Acta Neurochirurgica, Vol. 152, pp. 173-176. http://doi.org/10.1007/s00701-009-0394-2

Bogu, V.P., Kumar, Y.R., Kumar and Khanara, A. (2017), "Modelling and structural analysis of skull/cranial implant: beyond mid-line deformities", Acta of Bioengineering and Biomechanics Original paper, Vol. 19 No. 1, pp. 125-131. http://doi.org/10.5277/ABB-00547-2016-04

Breik, O., Mahindu, A., Moore, M.H., Molloy, C.J., Santoreneos, S. and David, D.J. (2016), “Apert syndrome: Surgical outcomes and perspectives”, Journal of Cranio-Maxillo-Facial Surgery, Vol. 44, pp. 1238-1245. http://doi.org/10.1016/j.jcms.2016.06.001

Brunzini, A., Mandolini, M., Manieri, S., Germani, M., Mazzoli, A., Pagnoni, M., Iannetti, G. and Modugno, A. (2017), "Orbital wall reconstruction by selective laser sintered mould", Proceedings of the IASTED International Conference in Biomedical Engineering (BioMed 2017), February 20-21, 2017, Innsbruck, Austria, pp. 260-264. http://doi.org/10.2316/P.2017.852-045

Carr, J.C., Fright, W.R. and Beatson, R.K. (1997), "Surface interpolation with radial basis functions for medical imaging”, IEEE Transactions on Medical Imaging, Vol. 16, pp. 96-107. http://doi.org 10.1109/42.552059

Dean, D. and Min, K.J. (2003), "Deformable templates for preoperative computer-aided design and fabrication of large cranial implants", International Congress Series, pp. 710-715. https://doi.org/10.1016/S05315131(03)00514-4

Driessen, C., van Veelen, M. L. C., Joosten, K. F. M., Versnel, S. L., van Nieuwenhoven, C. A. E., Wolvius, B., Bredero-Boelhouwer, H. H., Arnaud, E. and Mathijssen, I. M. J. (2017), “Apert syndrome: the Paris and Rotterdam philosophy", Expert Opinion on Orphan Drugs, Vol. 5 No. 7, pp. 599-605. http://doi.org/10.1080/21678707.2017.1335195

Drstvensek, I., Hren, N.I., Strojnik, T., Brajlih, T., Valentan, B., Pogacar, V. and Hartner, T.Z. (2008), "Applications of Rapid Prototyping in Cranio-Maxilofacial Surgery Procedures", International Journal of Biology and Biomedical Engineering, Vol. 2 No. 1, pp. 29-38.

Dumbrigue, H.B., Arcuri, M.R., LaVelle, W.E. and Ceynar, K.J. (1998), "Fabrication procedure for cranial prostheses", Journal of Prosthetic Dentistry, Vol. 79 No. 2, pp. 229-31. https://doi.org/10.1016/S00223913(98)70222-7

Farkas (1994), "Anthropometry of the head and face", Raven, second edition.

Gunz, P. and Mitteroecker, P. (2013), "Semilandmarks: a method for quantifying curves and surfaces", Hystrix, the Italian Journal of Mammalogy, Vol. 24 No. 1, pp. 103-109. https://doi.org/10.4404/hystrix-24.1-6292

Kai, C.C. Meng, C.S., Ching, L.S., Teik, L.S. and Aung, S.C. (2000), "Facial prosthetic model fabrication using rapid prototyping tools", Integrated Manufacturing Systems, Vol. 11 No. 1, pp. 42-53. https://doi.org/10.1108/09576060010303668

Karageorgiou, V. and Kaplan, D. (2005), "Porosity of 3D biomaterial scaffolds and osteogenesis", Biomaterials, Vol. 26, pp. 5474-5491. http://doi.org/10.1016/j.biomaterials.2005.02.002

Milovanović, J. and Trajanović, M. (2007), "Medical applications of rapid prototyping”, Mechanical Engineering Vol. 5 No. 1, pp. 79-85

Mandolini, M., Brunzini, A., Germani, M., Manieri, S., Mazzoli, A. and Pagnoni, M. (2019), "Selective laser sintered mould for orbital cavity reconstruction", Rapid Prototyping Journal, Vol. 25 No.1, pp. 95-103. http://doi.org/10.1108/RPJ-05-2017-0098

Moiduddin, K., Al-Ahmari, A., Abouel Nasr, E.S., Al Kindi, M., Ramalingam, S. and Kamrani, A. (2016), "Comparing 3-Dimensional Virtual Reconstruction methods in Customized Implants", International Journal of Advanced Biotechnology and Research, Vol. 7 No. 1, pp. 323-331.

Muschler, G. F., Nakamoto, C. and Griffith, L. G. (2004), "Engineering Principles of Clinical Cell-Based Tissue Engineering", The Journal of Bone and Joint Surger, Vol. 86, pp. 1541-1558.

Park, D.K., Song, I., Lee, J.H. and You, Y.J. (2013), "Forehead Augmentation with a Methyl Methacrylate Onlay Implant Using an Injection-Molding Technique", Archives of Plastic Surgery, Vol. 40 No. 5, pp. 597-602. https://doi.org/10.5999/aps.2013.40.5.597

Shah, A.M., Jung, H. and Skirboll, S. (2014), "Materials used in cranioplasty: a history and analysis", Neurosurg Focus, Vol. 36 No. 4, pp. 1-7. http://doi.org/10.3171/2014.2.FOCUS13561.

Wung, T., Engelhardt, M., Fieten, L., Popovic, A. and Radermacher, K. (2006), “Anatomically Constrained Deformation for Design of Cranial Implant: Methodology and Validation”, Medical Image Computing and Computer-Assisted Intervention, Vol. 9 No. 1, pp. 9-16. https://doi.org/10.1007/11866565_2 